\title{
Treatment of irreparable rotator cuff tears
}

\author{
M. Michael Khair • Lawrence V. Gulotta
}

Published online: 1 September 2011

(C) Springer Science+Business Media, LLC 2011

\begin{abstract}
Irreparable rotator cuff tears are a challenging problem for patients and surgeons. There are several treatment options, but deciding the correct one for each patient can be difficult. Treatment options include physical therapy, arthroscopy, muscle transfers, reverse shoulder arthroplasty, and hemiarthroplasty. An understanding of the patient's chief complaint and their functional ability to elevate their arm above horizontal should guide the treatment. This article reviews the current literature on various treatment options for irreparable rotator cuff tears, then outlines an algorithm for determining treatment.
\end{abstract}

Keywords Massive rotator cuff tears · Irreparable rotator cuff tears · Margin convergence · Physical therapy · Shoulder hemiarthroplasty Latissimus dorsi transfer Pectoralis major transfer- Reverse shoulder arthroplasty

\section{Introduction}

Rotator cuff tears are a common source of pain and disability in the elderly population. The natural history of rotator cuff tears is progression over time, with some tears developing tendon retraction and fatty infiltration of the muscle. When this occurs, it becomes technically difficult

M. M. Khair

Resident in Orthopedic Surgery, Hospital for Special Surgery, 535 E 70th Street,

New York, NY 10021, USA

\section{V. Gulotta $(\bowtie)$}

Assistant Attending Surgeon, Hospital for Special Surgery, 535 E 70th Street,

New York, NY 10021, USA

e-mail: GulottaL@hss.edu to repair the tendon back to its anatomic footprint. Even when mobilization and repair is achieved, a high radiographic failure rate often occurs when the tendon and muscle are severely degenerated. Many surgeons have concluded that these unpredictable results do not justify the morbidity of sling immobilization, several months of physical therapy, and missed time from work, that are all necessary following a rotator cuff repair. Therefore, alternative treatments have been introduced to assist patients with irreparable rotator cuff tears.

Clinical factors that point towards an irreparable rotator cuff tear include a long duration of symptoms, weakness in external rotation, an acromiohumeral interval (AHI) of less than $6 \mathrm{~mm}$ on the true anteroposterior radiograph, tendon retraction to the glenoid, and fatty infiltration of the rotator cuff musculature $\geq 50 \%$ on computed tomography (CT) or magnetic resonance imaging (MRI). When these factors are present, there is a high likelihood that the tendons will not heal even if mobilization and repair is feasible. Therefore, irreparable rotator cuff tears are ones that should not be repaired for lack of healing potential, as opposed to those that cannot be repaired due to technical limitations. While most irreparable rotator cuff tears can also be considered massive tears, not all massive tears are irreparable. Many massive rotator cuff tears lack the clinical and radiographic features outlined above, and are therefore amenable to repair.

In the setting of an irreparable rotator cuff tear, the current options include physical therapy, arthroscopic debridement or partial repair with biceps tenotomy and possible suprascapular nerve release, muscle transfers, reverse total shoulder arthroplasty, and hemiarthroplasty. The purpose of this paper is to review the literature to determine the indications and expected outcomes for each intervention in the setting of an irreparable rotator cuff tear. This information is useful in determining a treatment algorithm for this challenging clinical situation. 


\section{Non-operative treatment}

In the absence of anterosuperior escape, physical therapy with or without steroid injections should be the first line of treatment. Anterior deltoid strengthening has been shown to achieve elevation even in patients who originally present with pseudoparalysis, or elevation below $90^{\circ}$. Levy et al. prospectively assessed 17 patients with clinically and radiographically diagnosed irreparable rotator cuff tears that underwent an anterior deltoid training program [1•]. By 9 months, the mean Constant score improved from 26 to 63 , and the forward flexion improved from $40^{\circ}$ to $160^{\circ}$. The authors concluded that in $90 \%$ of patients, the effect of recruiting the anterior deltoid to stabilize the shoulder was sufficient to improve pain and function adequately enough to avoid surgery.

Other studies have also shown good results with physical therapy for patients with irreparable rotator cuff tears. Ainsworth et al. evaluated a multimodal physical therapy program that emphasized patient education, posture correction, re-education of muscle recruitment, strengthening, stretching, improved proprioception, and adaptation [2]. Ten patients participated in this program and by 12 weeks the mean Oxford Shoulder Disability Questionnaire (OSDQ) score improved by 9 points, and the SF-36 scores improved by 22 points. Zingg et al. evaluated the long-term outcomes of patients with irreparable tears that were treated conservatively [3]. They retrospectively evaluated 19 patients at a mean follow-up of 48 months. Over this time, glenohumeral osteoarthritis progressed as did the size of the tendon tears and the measured fatty infiltration. Despite this, the authors found that functional outcome scores remained acceptable, with a mean absolute Constant score to be 69 , a relative Constant score to be 83 , and a mean subjective shoulder value of 68. Pain averaged 11.5 on a $0-15$ point visual analog scale, where a score of 15 meant no pain. The authors concluded that nonoperatively treated massive rotator cuff tears in moderately symptomatic patients could maintain satisfactory shoulder function despite the progression of degenerative pathology. While this study shows that there are some patients who respond well to nonoperative treatment, the retrospective nature of it does not allow comparisons to be made to patients who failed conservative treatment over the course of 48 months.

\section{Arthroscopy}

There is some controversy over the role of arthroscopy in the management of irreparable rotator cuff tears. Arthroscopic debridement, partial repair with margin convergence, biceps tenotomy or tenodesis, and more recently supra- scapular nerve release have all been described as potential treatments. The literature would suggest that they are effective at alleviating pain, but have little effect on strength.

Several authors have studied the results of arthroscopic debridement. Rockwood et al. performed an acromioplasty, subacromial decompression, and debridement of massive infraspinatus and supraspinatus tears in 57 patients [4]. At an average of 6.5 years, 32 of the 53 shoulders had an excellent UCLA score, 12 were good, 4 were fair and 5 were poor. The average active elevation of the shoulder increased from $105^{\circ}$ to $140^{\circ}$. The average pain score went from 1 (constant and unbearable, frequent use of strong medicine) to 8.9 (occasional to none). Gartsman et al. reported significant pain relief in 33 patients who underwent arthroscopic debridement for a massive irreparable tears [5]. However, good early results may not be durable over time. Zvijac et al. compared the results at 2 and 4 years of 24 patients who underwent debridement [6]. They found that significantly less $(16 \%)$ patients were still doing satisfactory at 4 years when compared to 2 years. Pain and function had decreased while strength and range of motion were relatively unaffected. The authors found that among the patients, those with massive cuff tears did worse. As a result of their study, the authors concluded that debridement and decompression can give patients some satisfaction in the short term (1-3 years) but that over time, these favorable results deteriorate.

In a select group of patients, those with good remaining tissue quality, partial arthroscopic repair of massive rotator cuff tears has been reported to have good outcomes. Surgeons argue that in place of completely repairing a massive tear, repairing part can restore a stable fulcrum at the glenohumeral joint and achieve increased function. For example, in cases where it is not feasible to repair both the infraspinatus and supraspinatus back to bone, repairing the infraspinatus alone can improve external rotation strength and functional outcomes. Similarly, using the technique of margin convergence, sutures can be placed in the remaining cuff, that while not repairing to bone, increase the mechanical advantage of the rotator cuff and can restore some biomechanical balance to the shoulder joint $[7,8]$.

The role of the long head of the biceps in the shoulder continues to be debated, though consensus is mounting that it offers little functional benefit and functions solely as a pain generator. Walch et al. performed 307 biceps tendon tenotomies in patients with full-thickness rotator cuff tears that were considered irreparable [9]. At an average of 57 months follow-up, the mean Constant score increased from 48.4 points to 67.6 points and they reported that $87 \%$ of the patients were satisfied with the results of the surgery. The arthroscopic tenotomy, while helping with functional 
outcomes and patients' pain, did nothing to alter the course of glenohumeral arthritis.

The role that neuropathy of the suprascapular nerve (SSN) in the pain and weakness associated with massive rotator cuff tears remains unclear. Massive posterosuperior cuff tears can place traction on the SSN as the rotator cuff muscles retract. Several authors, using electrodiagnostic studies, have documented SSN neuropathy in patients with massive cuff tears. Some of these studies also documented partial or complete recovery following partial or complete repair. Mallon et al. prospectively studied eight patients presenting with massive rotator cuff tears that showed retraction and fatty infiltration of the supraspinatus muscle on MRI [10]. The eight patients all had suprascapular neuropathy as diagnosed by electromyelography (EMG). Four of the eight patients underwent partial repair using margin convergence with a mini-open approach. Half of the surgical patients underwent a repeat EMG 6 months postoperatively. In both cases, the EMG showed that the suprascapular nerve has significant renervation potential. Vad et al. studied did nerve conduction studies on 25 patients with full thickness rotator cuff tears [11]. They found 7 of the 25 to have abnormal EMG studies. The axillary nerve was affected in four of the seven patients while the suprascapular nerve was affected in two of seven. The overall prevalence of a peripheral neuropathy in patients with a full thickness rotator cuff tear was found to be $28 \%$. Greater levels of atrophy were associated with a higher likelihood of finding a neuropathy. Costouros et al. identified 26 patients with massive rotator cuff tears [12]. Using EMG and nerve conduction velocity (NCV) studies, 14 of those patients with massive cuff tears were found to have a concomitant peripheral neuropathy. Seven of the fourteen were found to have an isolated suprascapular neuropathy. Four were found to have an axillary neuropathy, two had an upper trunk brachial plexus injury and one had a cervical radiculopathy. Six of the seven patients with the isolated SSN were treated with either complete or partial repair. Of these patients, 6 months later, repeat EMG/ NCV showed partial or full recovery of the SSN.

\section{Latissimus dorsi transfer}

Patients that present with a primary complaint of weakness in the setting of an irreparable rotator cuff tear are not candidates for arthroscopic procedures. Transfer of the latissimus dorsi can help restore external rotation, and some degree of elevation in patients with posterosuperior rotator cuff tears. Gerber et al. evaluated the results of latissumus dorsi transfers in 63 patients at an average follow-up of 53 months [21]. They found that the Subjective Shoulder Score increased from $28 \%$ to
$66 \%$, Constant scores increased from $55 \%$ to $73 \%$, and forward flexion increased from a mean value of $104^{\circ}$ to $123^{\circ}$. Most importantly, they found that the patients that had the best outcomes are those that had an intact subscapularis, and those that were able to elevate to horizontal prior to the surgery.

Other authors have also had success with latissumus dorsi transfers. Birmingham and Neviaser reviewed 18 patients who were referred to their institution for failed repair of massive posterosuperior rotator cuff tears [22]. None of the patients had evidence of anterosuperior escape. They found that the average ASES score increased from 43 pre-operatively to 61 post-operatively, pain scores improved from 59 to 22 , active elevation increased from 56 to an average of $137^{\circ}$, and active external rotation improved from $31^{\circ}$ to $45^{\circ}$. Iannotti et al. published the results of latissimus dorsi transfer in 14 patients who were for 24 months [23]. The average PENN score increased from 40 points to 66 points. Overall, nine patients were satisfied with the results of the surgery and five patients were not. Average active forward flexion increased from $100^{\circ}$ to $119^{\circ}$ overall, however, in patients who were dissatisfied with their surgery, it decreased from $64^{\circ}$ to $58^{\circ}$. External rotation in $0^{\circ}$ of abduction increased from $23^{\circ}$ to $32^{\circ}$ and at $90^{\circ}$ of abduction improved from $24^{\circ}$ to $50^{\circ}$. The average postoperative isometric external rotation strength increased in all patients. Warner and Parsons compared the outcomes of patients who underwent a latissimus dorsi transfer as a salvage procedure to the outcomes of patients who underwent latissimus dorsi transfer as a primary reconstruction for what was considered an irreparable rotator cuff tear [24]. The mean time to follow-up was 25 months in the group receiving a salvage reconstruction transfer and 29 months in those receiving a primary reconstruction. Postoperative forward flexion in the primary group measured $122^{\circ}$ with a relative gain of $60^{\circ}$ between pre and postoperative testing. In the group with transfer as a salvage operation, the gain was on average $43^{\circ}$, for a final average elevation of $105^{\circ}$. Active external rotation was similar between the two groups. The post-operative Constant score was $69 \%$ in the primary group versus $52 \%$ in the revision group. The relative gain in the Constant score was 33\% in the primary repair group versus $16 \%$ in the revision group. Fatty degeneration and deltoid detachment were also significantly associated with inferior outcomes.

\section{Pectoralis major transfer}

The transfer of the pectoralis major tendon has been used as a treatment option for patients with irreparable tears of the subscapularis. When accurately transferred, the pectoralis major tendon can help patients regain 
internal rotation of the shoulder. Jost et al. followed 28 patients who underwent a pectoralis major transfer for an irreparable tear of the subscapularis tendon [25]. Twelve tears were isolated subscapularis tears and 18 were associated with tears of the supraspinatus or infraspinatus. Patients were seen at an average of 32 months after the operation. The mean Constant score increased from $47 \%$ pre3-operatively to $70 \%$ at follow-up. Specifically, the scores for pain, ADLs, forward flexion and abduction strength were significantly increased. Most patients were satisfied with the surgery, those that did less favorable had associated irreparable supraspinatus tears. The authors concluded that pectoralis major tendon transfers can work well for isolated irreparable subscapularis tears but might not be indicated when there are ipsilateral irreparable supraspinatus tears as well.

Galatz et al. reported on the use of pectoralis major muscle transfer for the treatment of anterior-superior shoulder subluxation in massive rotator cuff insufficiency [26]. They followed 14 patients who underwent subcorocoid pectoralis major muscle transfer. At 17.5 months of follow-up, results were satisfactory in 11 patients and unsatisfactory in three. ASES functional scores increased from 27.2 to a mean of 47.7. Active forward flexion increased from $28.4^{\circ}$ to $60^{\circ}$. Thirteen of the 14 patients had improved humeral head containment. The authors concluded that pectoralis major tendon transfer is a viable option for patients with anterior-superior subluxation due to massive rotator cuff insufficiency.

\section{Reverse total shoulder arthroplasty}

Patients that have true pseudoparalysis of the shoulder are not candidates for arthroscopic procedures or tendon transfers. The only reliable procedure to improve elevation is a reverse total shoulder arthroplasty. While originally the indications for this procedure were limited to those with cuff tear arthropathy, they have expanded to include patients with massive, irreparable rotator cuff tears and the inability to elevate the arm.

Wall et al. have reported the results of the early French experience with the reverse total shoulder in 168 patients who were followed for an average of 39.9 months [18••]. Thirty-four of these were indicated for surgery because of a massive rotator cuff tear in the absence of arthritis [18••]. Within that group, the total Constant score improved from 27.8 pre-operatively to 63.4 at the time of final follow-up. Specifically, pain improved from 3.8 to 12.2 , activity from 5.6 to 15 , mobility from 16.9 to 28.4 , and, strength from 1.5 to 7.8. Range of motion in the massive cuff tear group increased from $94^{\circ}$ to $143^{\circ}$ in elevation, decreased from $14^{\circ}$ to $8^{\circ}$ in external rotation. Frankle et al. examined 60 shoulders over a minimum of 2 years with rotator cuff deficiency and glenohumeral arthritis that were treated with the Reverse Shoulder Prosthesis [17]. The average ASES score rose from 34 pre-operatively to 68.2 post-operatively and active forward flexion improved from an average of $55^{\circ}$ to $105.1^{\circ}$ after the surgery. Werner et al. reported the results of 58 consecutive patient with moderate to severe shoulder pain and less than $90^{\circ}$ of active shoulder anterior elevation due to an irreparable rotator cuff tear who were treated with the reverse and followed for 38 months [19]. Of the 58 patients, 17 had rotator cuff tear arthropathy with no previous operation, the other 41 were revision procedures. Concerning the 17 which are most pertinent to this review, the subjective shoulder value increased from 19 to 59 and the relative Constant score increased from 35 to 72 . Forward flexion increased from $43^{\circ}$ to $103^{\circ}$ and active abduction increased from $39^{\circ}$ to $84^{\circ}$. Like previous studies, the authors of this study concluded that the reverse shoulder prosthesis has the ability to improve the condition of patients with shoulder dysfunction at least in the short term.

Boileau et al. studied the results of the implantation of the Grammont reverse shoulder prosthesis in 2005 [20]. Of the 45 patients the authors followed, 21 were indicated for surgery based on the presence of a massive cuff tear and arthrosis (CTA). Range of motion increased in the CTA group between pre-operative measurements and those taken at follow-up. Active anterior elevation increased from $53^{\circ}$ to $123^{\circ}$. External rotation increased from $9^{\circ}$ to $14^{\circ}$. The Constant score in the CTA group increased from 18 to 66 . The CTA patients also fared better on the ASES than the other groups the authors studied. The overall conclusion was that the Grammont reverse shoulder prosthesis can improve shoulder function and restore active elevation in patients with cuff-deficient shoulders.

\section{Hemiarthroplasty}

Hemiarthroplasty is a possible treatment option for a patient with irreparable rotator cuff arthropathy. It is an option best reserved for a patient with modest functional goals who has an intact subscapularis tendon and a preserved axial force couple [13]. In the author's practice, hemiarthroplasty is indicated for patients with an irreparable rotator cuff tear, glenohumeral arthritis, and the ability to elevate the arm (ie, not pseudoparalytic).

Williams et al. reviewed 21 humeral hemiarthroplasties done using the Neer prosthesis between the years of 1974 to 1990 in rotator cuff deficient shoulders [14]. Eighteen of the 21 shoulders obtained satisfactory results as defined by Neer et al.'s limited goals criteria. Using the modified grading 
system for patients with a complete cuff, there were no excellent results, 14 satisfactory results, and 7 unsatisfactory results. Twelve shoulders had a post-operative pain score of zero. Active flexion increased on average from $70^{\circ}$ preoperatively to $120^{\circ}$, and external rotation increased from $27^{\circ}$ to $46^{\circ}$. The authors concluded that hemiarthroplasty in a rotator cuff deficient shoulder is inferior to shoulder arthroplasty in patients with glenohumeral arthritis and an intact cuff. Sanchez-Sotelo et al. retrospectively followed 33 shoulders with glenohumeral arthritis and massive rotator cuff deficiency that were treated with a hemiarthroplasty [16]. Their average time of follow-up was 5 years. They found that the mean pain score decreased following the surgery and their active elevation increased from an average of $72^{\circ}$ to $91^{\circ}$. No difference in strength was detected in shoulder abduction or flexion. The overall result was graded as successful in 22 shoulders and unsuccessful in 11 . Zuckerman et al. performed a retrospective review examining 15 cases of shoulder hemiarthroplasty for rotator cuff arthropathy [15]. The average forward flexion increased from $69^{\circ}$ to $86^{\circ}$ and external rotation increased from $15^{\circ}$ to $29^{\circ}$. All patients had an increase in their ability to do activities of daily living. Thirteen of fifteen shoulders expressed an overall satisfaction with the outcome of their surgery and all but 1 patient expressed significant pain relief. The average increase on the UCLA Rating scale was 11 to 22 points. The authors of the study concluded that favorable clinical results can be achieved with hemiarthroplasty in the shoulder with massive rotator cuff deficiency.

Fig. 1 Treatment algorithm for irreparable rotator cuff tears

\section{Conclusions}

Irreparable rotator cuff tears are a challenging problem. There are several treatment options, but determining the correct treatment for each patient can be difficult. The author's treatment algorithm is outlined in Fig. 1. Patients without anterosuperior escape should initially be treated with physical therapy for anterior deltoid training. In patients who fail physical therapy, or have escape, it is important to determine if the patient's primary complaint is pain or weakness, and to determine if the patients is able to elevate their arm above horizontal on exam. If a patient has pain, and is not able to elevate their arm, then the authors have found an injection of local anesthesia and steroids can be helpful in determining true pseudoparalysis from pain inhibition. Patients with pain who are able to elevate either before or after an injection are excellent candidates for arthroscopic debridement or partial repair, biceps tenotomy, and possibly suprascapular nerve release. Patients who complain of weakness in external rotation, but have elevation to horizontal and an intact subscapularis are ideally suited for a latissumus transfer. Those with an irreparable subscapularis, but an intact superior cuff without escape are candidates for a pectoralis major transfer. Patients with arthritis on their radiographs in the setting of an irreparable rotator cuff tear, but are still able to elevate, are excellent candidates for hemiarthroplasty is indicated. Patients with true psuedoparalysis are best treated with the reverse shoulder prosthesis.

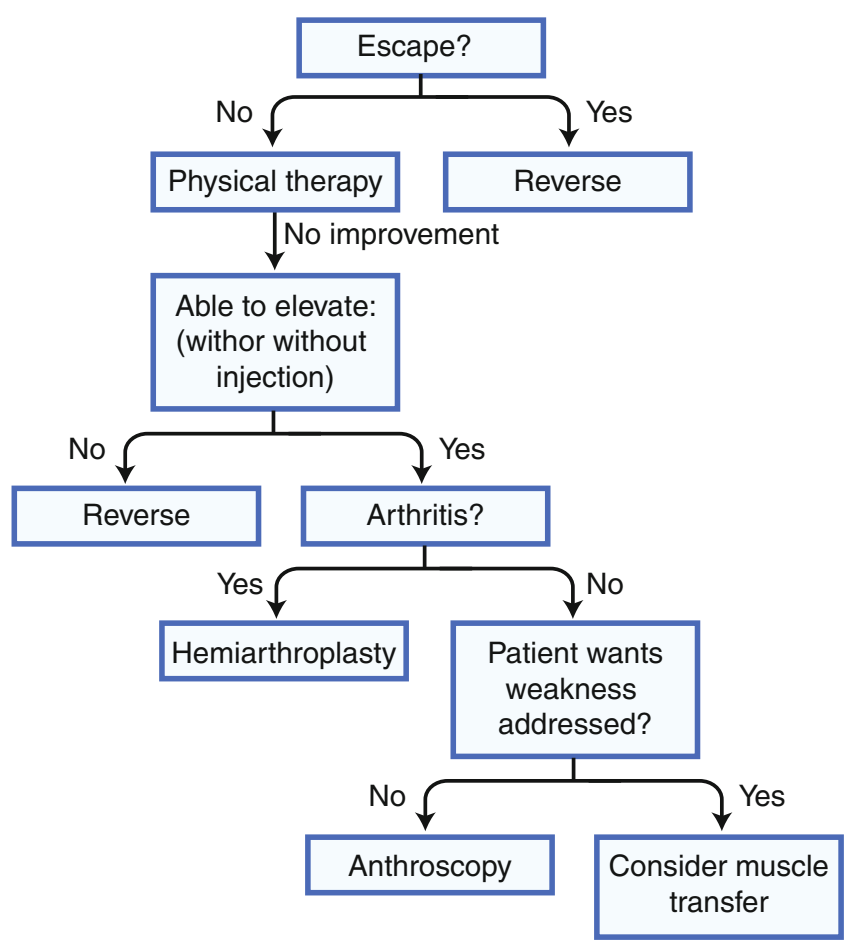


Disclosure No conflicts of interest relevant to this article were reported.

\section{References}

Papers of particular interest, published recently, have been highlighted as:

- Of importance

-• Of major importance

1. - Levy O, Mullett H, Roberts S, Copeland S. The role of anterior deltoid reeducation in patients with massive irreparable degenerative rotator cuff tears. J Shoulder Elbow Surg. 2008;17(6):863-70. This study showed good results with anterior deltoid training for patients with massive, irreparable rotator cuff tears.

2. Ainsworth R. Physiotherapy rehabilitation in patients with massive, irreparable rotator cuff tears. Musculoskeletal Care. 2006;4(3):140-51.

3. Zingg PO, Jost B, Sukthankar A, Buhler M, Pfirrmann CW, Gerber C. Clinical and structural outcomes of nonoperative management of massive rotator cuff tears. J Bone Joint Surg Am. 2007;89(9):1928-34.

4. Rockwood Jr CA, Williams Jr GR, Burkhead Jr WZ. Debridement of degenerative, irreparable lesions of the rotator cuff. J Bone Joint Surg Am. 1995;77(6):857-66.

5. Gartsman GM. Massive, irreparable tears of the rotator cuff. Results of operative debridement and subacromial decompression. J Bone Joint Surg Am. 1997;79(5):715-21.

6. Zvijac JE, Levy HJ, Lemak LJ. Arthroscopic subacromial decompression in the treatment of full thickness rotator cuff tears: a 3- to 6-year follow-up. Arthroscopy. 1994;10(5):518-23.

7. Burkhart SS. The principle of margin convergence in rotator cuff repair as a means of strain reduction at the tear margin. Ann Biomed Eng. 2004;32(1):166-70.

8. Burkhart SS, Athanasiou KA, Wirth MA. Margin convergence: a method of reducing strain in massive rotator cuff tears. Arthroscopy. 1996;12(3):335-8.

9. Walch G, Edwards TB, Boulahia A, Nove-Josserand L, Neyton L, Szabo I. Arthroscopic tenotomy of the long head of the biceps in the treatment of rotator cuff tears: clinical and radiographic results of 307 cases. J Shoulder Elbow Surg. 2005;14(3):238-46.

10. Mallon WJ, Wilson RJ, Basamania CJ. The association of suprascapular neuropathy with massive rotator cuff tears: a preliminary report. J Shoulder Elbow Surg. 2006;15(4):395-8.

11. Vad VB, Southern D, Warren RF, Altchek DW, Dines D. Prevalence of peripheral neurologic injuries in rotator cuff tears with atrophy. J Shoulder Elbow Surg. 2003;12(4):333-6.

12. Costouros JG, Porramatikul M, Lie DT, Warner JJ. Reversal of suprascapular neuropathy following arthroscopic repair of massive supraspinatus and infraspinatus rotator cuff tears. Arthroscopy. 2007;23(11):1152-61.

13. Bedi A, Dines J, Warren RF, Dines DM. Massive tears of the rotator cuff. J Bone Joint Surg Am. 2010;92(9):1894-908.

14. Williams Jr GR, Rockwood Jr CA. Hemiarthroplasty in rotator cuff-deficient shoulders. J Shoulder Elbow Surg. 1996;5(5):362-7.

15. Zuckerman JD, Scott AJ, Gallagher MA. Hemiarthroplasty for cuff tear arthropathy. J Shoulder Elbow Surg. 2000;9(3):169-72.

16. Sanchez-Sotelo J, Cofield RH, Rowland CM. Shoulder hemiarthroplasty for glenohumeral arthritis associated with severe rotator cuff deficiency. J Bone Joint Surg Am. 2001;83-A(12):1814 22.

17. Frankle M, Levy JC, Pupello D, Siegal S, Saleem A, Mighell M, et al. The reverse shoulder prosthesis for glenohumeral arthritis associated with severe rotator cuff deficiency. a minimum twoyear follow-up study of sixty patients surgical technique. J Bone Joint Surg Am. 2006;88(Suppl 1 Pt 2):178-90.

18. •• Wall B, Nove-Josserand L, O'Connor DP, Edwards TB, Walch G. Reverse total shoulder arthroplasty: a review of results according to etiology. J Bone Joint Surg Am 2007;89(7):147685. Comprehensive review of the French experience with the reverse total shoulder.

19. Werner CM, Steinmann PA, Gilbart M, Gerber C. Treatment of painful pseudoparesis due to irreparable rotator cuff dysfunction with the Delta III reverse-ball-and-socket total shoulder prosthesis. J Bone Joint Surg Am. 2005;87(7):1476-86.

20. Boileau P, Watkinson D, Hatzidakis AM, Hovorka I. Neer Award 2005: The Grammont reverse shoulder prosthesis: results in cuff tear arthritis, fracture sequelae, and revision arthroplasty. J Shoulder Elbow Surg. 2006;15(5):527-40.

21. Gerber C, Maquieira G, Espinosa N. Latissimus dorsi transfer for the treatment of irreparable rotator cuff tears. J Bone Joint Surg Am. 2006;88(1):113-20.

22. Birmingham PM, Neviaser RJ. Outcome of latissimus dorsi transfer as a salvage procedure for failed rotator cuff repair with loss of elevation. J Shoulder Elbow Surg. 2008;17(6):871-4.

23. Iannotti JP, Hennigan S, Herzog R, Kella S, Kelley M, Leggin B, et al. Latissimus dorsi tendon transfer for irreparable posterosuperior rotator cuff tears. Factors affecting outcome. J Bone Joint Surg Am. 2006;88(2):342-8.

24. Warner JJ, Parsons 4th IM. Latissimus dorsi tendon transfer: a comparative analysis of primary and salvage reconstruction of massive, irreparable rotator cuff tears. J Shoulder Elbow Surg. 2001;10:514-21.

25. Jost B, Puskas GJ, Lustenberger A, Gerber C. Outcome of pectoralis major transfer for the treatment of irreparable subscapularis tears. J Bone Joint Surg Am. 2003;85-A(10):1944-51.

26. Galatz LM, Connor PM, Calfee RP, Hsu JC, Yamaguchi K. Pectoralis major transfer for anterior-superior subluxation in massive rotator cuff insufficiency. J Shoulder Elbow Surg. 2003;12(1):1-5. 